\title{
Context effects on conditioning, extinction, and reinstatement in an appetitive conditioning preparation
}

\author{
MARK E. BOUTON and CHARLES A. PECK \\ University of Vermont, Burlington, Vermont
}

\begin{abstract}
Three experiments with rat subjects examined the effects of contextual stimuli on performance in appetitive conditioning. A 10-sec tone conditioned stimulus (CS) was paired with a food-pellet unconditioned stimulus (US); conditioning was indexed by the observation of headjerking, a response of the rat to auditory stimuli associated with food. In Experiment 1, a context switch following initial conditioning did not affect conditioned responding to the tone; however, when the response was extinguished in the different context, a return to the original conditioning context "renewed" extinguished responding. These results were replicated in Experiments 2 and 3 after equating exposure to the two contexts (Experiment 2) and massing the conditioning and extinction trials (Experiment 3). The results of Experiment 1 also demonstrated that separate exposure to the US following extinction reinstates extinguished responding to the tone; this effect was further shown to depend at least partly on presenting the US in the context in which testing is to occur (Experiments 2 and 3). Overall, the results are consistent with previous data from aversive conditioning procedures. In either appetitive or aversive conditioning, the context may be especially important in affecting performance after extinction.
\end{abstract}

The role of contextual or background stimuli in Pavlovian conditioning has been studied fairly extensively using the conditioned suppression method. In that method, conditioned stimuli (CSs) are paired with footshock unconditioned stimuli (USs), and the degree to which they suppress an ongoing baseline behavior provides an index of the strength of conditioning. It seems clear that contextual or background stimuli are often important in determining performance in this paradigm. For example, if CS-US pairings are given in one context (Context A), and extinction is then produced by CS-alone presentations in another context (Context B), fear of the CS is "renewed" if the CS is tested back in Context A (Bouton \& Bolles, 1979a; Bouton \& King, 1983; Hanford, Mulvaney \& Kelfer, 1980; Lovibond, Preston, \& Mackintosh, 1984) or in a third context (Bouton \& Bolles, 1979a; see also Bouton \& Swartzentruber, 1986, Experiment 1). In addition, if the animal is exposed to the US after extinction so as to condition excitation to the context, contextual conditioning can "reinstate" fear of the extinguished CS (Bouton, 1984; Bouton \& Bolles, 1979b; Bouton \& King, 1983). Renewal and reinstatement both suggest that the response evoked by the CS in conditioned suppression can depend substantially on the context.

Interestingly, extinguished CSs are especially sensitive to these types of manipulation of the context. In contrast

This research was supported by Grant BNS 86-07208 from the National Science Foundation. We thank Dale Swartzentruber for his comments on an earlier version of the manuscript. Correspondence may be addressed to Mark E. Bouton, Department of Psychology, University of Vermont, Burlington, VT 05405. to the clear effect of switching the context following extinction, a change of context following simple conditioning often fails to affect performance in response to the CS (Bouton \& King, 1983; Bouton \& Swartzentruber, 1986; Lovibond et al., 1984). Similarly, although conditioning of the context through separate US exposures clearly augments (i.e., reinstates) responding to an extinguished CS, the same treatment following conditioning typically fails to affect performance in response to a CS that is not extinguished (Bouton, 1984; Bouton \& King, 1986).

The relative sensitivity of the extinguished CS to context is analogous to that of an ambiguous word (e.g., bear; see Bouton, 1984, 1988; Bouton \& Bolles, 1985). For either type of stimulus, the context is especially important in determining the response it evokes (grizzly bear, bear fruit). This analogy suggests a number of implications for the theoretical analysis of both extinction and contextual control (see Bouton, 1988; Bouton \& Bolles, 1985). However, it is also possible that conditioned suppression engages a defensive motivational system which ensures that the rat will always behave cautiously in response to the CS. The ease with which fear can be renewed or reinstated after extinction, along with fear's ability to generalize across contexts after initial conditioning, may simply attest to the conservative nature of the defensive system.

The purpose of the present experiments was thus to assess the generality of some of the conditioned suppression data by examining similar effects in appetitive, rather than defensive, conditioning. Rats were exposed to tone-food-pellet pairings, and their conditioned response 
to the CS was videotaped and scored with methods based on those described by Holland (e.g., 1977, 1979). The context specificity of conditioning, extinction, and reinstatement was then examined. Although a role for context in appetitive conditioning has been suggested by previous experiments on the effects of CS or US preexposures on conditioning in rats (Channell \& Hall, 1983) and especially in pigeons (e.g., Balsam \& Schwartz, 1981; Grau \& Rescorla, 1984; Tomie, 1976), to our knowledge the present phenomena have never been addressed in appetitive conditioning. The present results suggest that they occur here much as they occur in aversive conditioning.

\section{EXPERIMENT 1}

In the first experiment, three groups received tone and food-pellet presentations in Context A. Two groups received tone-food pairings, while the third group (Group CTRL) received tone and food in an explicitly unpaired manner that was not expected to yield excitatory conditioning. In the next phase (extinction) all groups received exposure to the tone alone. One of the groups that had received initial tone-food pairings, Group AT, received extinction in Context $A$, the conditioning context; the other paired group, Group BT, received the same treatment in Context B. Comparing these groups during extinction would test whether a context switch following conditioning affects conditioned responding in this paradigm. (Half the animals in Group CTRL received the tone in Context A and the other half received it in B.) After the conclusion of extinction, all groups were given further tone-alone presentations in Context $\mathrm{A}$. In the conditioned suppression preparation, the return of Group BT to Context A for testing would renew extinguished responding to the tone.

The experiment also provided a preliminary study of reinstatement. After the completion of renewal testing, half the rats in Groups AT and BT were each reassigned to one of two new groups. One of these new groups, Group R (for reinstatement), received eight US exposures during a single session in the extinction context; $24 \mathrm{~h}$ later, further extinction tests of the tone were begun there. Group No-R (no reinstatement) received the same exposure to the apparatus prior to testing, but did not receive the US. Group CTRL, the group that had never had excitatory conditioning of the tone, received the same treatment as Group $\mathbf{R}$. If appetitive conditioning resembles conditioned suppression, one would expect more responding to the tone in Group $R$ than in either of the other groups during the final tests.

\section{Method}

Subjects

The subjects were 24 female Wistar rats bred at the University of Vermont. They were approximately 140 days old at the start of the experiment and were housed individually in standard stainless steel cages in a room maintained on a 12:12-h light:dark cycle. The experiment was conducted on consecutive days during the light por- tion of the cycle. The animals were food-deprived and maintained at $80 \%$ of their initial body weights throughout the experiment.

\section{Apparatus}

Two discriminably different contexts were provided by two sets of four boxes located in separate rooms of the laboratory. Each box measured $23 \times 13 \times 11 \mathrm{~cm}$; the walls and ceiling were constructed of clear acrylic plastic. Three of the walls were covered on the outside with black construction paper; the front wall $(23 \mathrm{~cm})$ and ceiling were transparent. A small $(1 \times 1 \mathrm{~cm})$ food cup protruded from the back wall $4.5 \mathrm{~cm}$ from the floor and $4.5 \mathrm{~cm}$ from the right wall. The animals were placed in the boxes through the ceiling. Illumination was provided by a 7.5-W red incandescent bulb mounted on the ceiling of the sound-attenuation chamber, $25 \mathrm{~cm}$ above the floor, where a speaker, the source of the CS, was also mounted.

Each of the four boxes in one room had a floor consisting of 3-mm bars staggered such that the odd-numbered bars were mounted $6 \mathrm{~mm}$ above the even-numbered bars; the distance between consecutive bars was $1.6 \mathrm{~cm}$. The bars were parallel to the right $(11-\mathrm{cm}$ ) wall, where a $1 \times 2.5 \mathrm{~cm}$ lever (nonfunctional in these experiments) was centered $4.5 \mathrm{~cm}$ above the floor. The three covered (therefore black) walls were lined with horizontal white stripes $1 \mathrm{~cm}$ wide, spaced $1 \mathrm{~cm}$ apart. Within each sound-attenuation chamber, a dish containing $10 \mathrm{ml}$ of $4 \%$ McCormick coconut extract solution provided a distinctive scent cue. The floors of the remaining four boxes consisted of 3-mm bars, spaced $1.8 \mathrm{~cm}$ apart, which were mounted diagonally with respect to the chamber walls. The three covered walls were left black. A $1 \times 2.5 \mathrm{~cm}$ nonfunctional lever was located near the center of the back wall, $5.5 \mathrm{~cm}$ above the floor and $8 \mathrm{~cm}$ from the food cup. In these boxes, a dish containing $10 \mathrm{ml}$ of a $2 \%$ McCormick anise extract solution provided the distinctive scent cue.

In each experimental room, the four sound-attenuation chambers were positioned together in a $2 \times 2$ arrangement. Double-paned clear acrylic plastic windows in the chamber fronts permitted a view of the boxes within. A single Panasonic low-light camera (Model WV1460) fitted with an 8-mm wide-angle lens monitored all four boxes simultaneously. The camera was mounted $3.1 \mathrm{~m}$ away from the sound-attenuation chambers and was angled approximately $30^{\circ}$ above the horizontal axis. Video signals from each room were recorded in a separate room by Panasonic video-cassette recorders (Model AG-2200) controlled by an AT\&T 6300 microcomputer. All recording and programming equipment was located in this other room.

The CS was a $10-\mathrm{sec}$ presentation of a $3000-\mathrm{Hz}$ tone $(80 \mathrm{~dB}$ re $20 \mu \mathrm{N} / \mathrm{m}^{2}$ [A]) provided by a single generator wired to identical speakers in each chamber. Background noise level was $65 \mathrm{~dB}$. The unconditioned stimulus consisted of two $45-\mathrm{mg}$ Noyes food pellets (Formula A) delivered $0.2 \mathrm{sec}$ apart.

\section{Procedure}

Contexts A and B refer to the conditioning context and the alternative context, respectively. The actual boxes were completely counterbalanced. All sessions were conducted on consecutive days and were $60 \mathrm{~min}$ in duration.

Magaxine training. The rats were initially trained to eat food pellets when they were delivered to the food cup in both contexts. On Day 1, the rats were trained in one box (counterbalanced); on Day 2, they received the same treatment in a box from the alternate context. Each day, the food magazine was first baited with four food pellets and, after a 15-min adaptation period, eight US presentations were delivered at intervals averaging $5.4 \mathrm{~min}$.

Conditioning. On Day 3, the rats were randomly assigned to one of three groups $(n=8)$. During each of the next 10 sessions, all groups received four CS and four US presentations in Context $A$. CSs occurred 15.8, 35.7, 45.9, and $51.6 \mathrm{~min}$ into the session on odd-numbered days, and $21.3,28.0,40.2$, and $59.5 \mathrm{~min}$ into the 
session on even-numbered days. For Groups AT and BT (for eventual extinction of the tone in Context $\mathrm{A}$ or $\mathrm{B}$, respectively), CS presentations terminated in the onset of the US. For Group CTRL, the CS and US were delivered in one of two explicitly unpaired sequences (TFFTFTTF or FTTFTFFT, where T denotes the CS and $F$ denotes the US), which alternated daily. The timing of the CSs within each session matched that of the other groups; USs were delivered at the times when the CS was delivered on the alternate day.

Extinction. On Day 14 all rats received 60 min of adaptation exposure to the context to be used in extinction; no CSs or USs were presented. [A similar adaptation session has been used in previous conditioned suppression research (e.g., Bouton \& King, 1983).] For the next 7 days, all groups received four presentations of the CS alone, 11.8, 26.3, 41.6, and $55.4 \mathrm{~min}$ into the session. For Group AT, extinction took place in Context A. For Group BT, extinction took place in Context B. For Group CTRL, half of the subjects (counterbalanced with respect to boxes) received equivalent tone exposure in Context $\mathrm{A}$ and the other half received it in Context B.

Renewal testing. On the next six sessions (beginning on Day 22, the day following the last extinction day), all animals were placed in Context $\mathbf{A}$, the context of conditioning, and given four daily nonreinforced tests of the CS. CSs were delivered on the schedule used during extinction. For Group AT, which had received extinction all along in Context $A$, these were merely continued extinction trials. Group BT, however, now received testing in the context where conditioning, but not extinction, had occurred.

Reinstatement. On the day following the last day of renewal testing, the animals from Groups AT and BT were divided into two new groups, Group R and Group No-R (for reinstatement and no reinstatement, respectively). Rats were reassigned so as to match the groups on performance during extinction and to counterbalance the boxes providing the extinction context, which was used as the test context during this phase. Half of the rats in each new group came from Group AT and half from Group BT. On the day following the end of renewal testing, Group $R$ received eight USs alone in the context in which extinction had occurred; USs were delivered at intervals averaging $5.4 \mathrm{~min}$. Group No-R spent an equal amount of time in its extinction context without any USs. Group CTRL received the same treatment (eight USs) as did Group $\mathbf{R}$. On each of the next 3 days, all animals received four nonreinforced tests of the $\mathrm{CS}$ in the context in which the reinstatement treatment took place (i.e., the extinction context). CSs were delivered on the schedule used during extinction.

Observation procedure. Beginning with the Ist day of conditioning, the behavior of the rats was scored by a single observer who viewed the videotapes after each session. Using a time-sampling method based on that of Holland (e.g., 1977), the observer scored each rat eight times during the 10-sec interval preceding the CS (the "pre-CS period") and eight times during the 10-sec CS on each trial. Observations were driven by a small light (recorded on the tape), which flashed at 1.25 -sec intervals, beginning $.25 \mathrm{sec}$ into the pre-CS period. The observer was free to rescore any trial as needed to ensure an accurate account of every trial.

We report the observations of one behavior, headjerk, which was defined as a short, rapid head movement independent of other movements of the body (cf. Holland, 1977, 1979). Although other behaviors were scored initially, headjerking has been our best overall indicator of conditioned appetitive excitation to the tone. The complete video record of the last session of conditioning, along with the first session of each of the other phases, was independently scored by a second observer who was blind to the experimental treatments. Interobserver agreement did not vary across sessions; it averaged $99 \%$ during the pre-CS and $88 \%$ during the CS periods.

Data analysis. Headjerk scores for the CS and pre-CS periods were created by computing the percentage of observations within each period that were scored as headjerk. Data from the CS periods were then evaluated by analyses of variance (ANOVAs); planned comparisons were made using the standard methods discussed by Howell (1982, pp. 280 ff.). Data from the corresponding pre-CS periods were analyzed with the distribution-free Kruskal-Wallis analysis of variance, because a large number of scores of zero made the distributions nonnormal. Throughout, the rejection criterion was set at $p<.05$.

\section{Results}

Conditioning

The acquisition of headjerking in response to the tone over sessions is shown at left in Figure 1. The figure and statistical analyses suggest that headjerking did occur in response to the tone in Groups AT and BT, but not in Group CTRL. (A tape malfunction destroyed half the data from each group on Sessions 1 and 2; Figure 1 presents

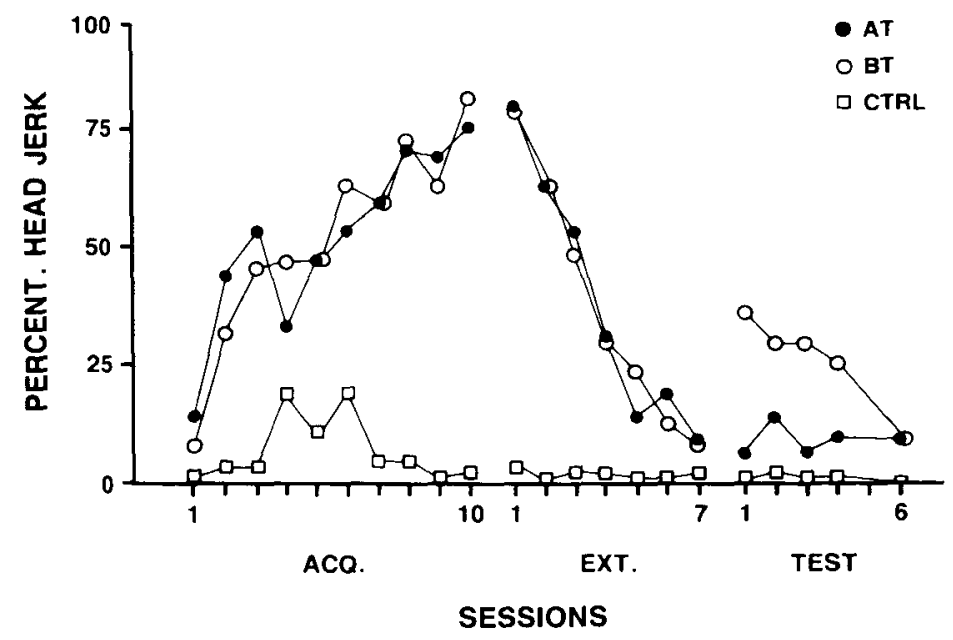

Figure 1. Mean percentage headjerking in response to the tone during the conditioning phase (left), extinction phase (middle), and renewal test phase (right) of Experiment 1. 
the surviving data, but Sessions 1 and 2 were excluded from the statistical analysis.) A group $\times$ session ANOVA revealed reliable main effects of group $[F(2,21)=40.68]$ and session $[F(7,147)=6.23]$, and a group $\times$ session interaction $[F(14,147)=5.36]$. Planned comparisons among the groups (collapsing over sessions) revealed significantly more headjerking in Groups AT and BT than in Group CTRL $[F \mathrm{~s}(1,21)>58.03]$, and no difference between Groups AT and BT $[F(1,21)<1]$.

Headjerking was confined primarily to the CS. During the pre-CS periods of the phase, Groups AT, BT, and CTRL had mean headjerk scores of $0.2 \%, 0.1 \%$, and $0.2 \%$, respectively, which did not differ significantly [Kruskal-Wallis $H(2)=0.47$ ].

\section{Extinction}

Headjerking in response to the CS during extinction is shown in the center of Figure 1. A separate group $\times$ session ANOVA on these data revealed significant main effects of group $[F(2,21)=57.66]$ and session $[F(6,126)$ $=64.20]$, and a group $\times$ session interaction $[F(12,126)$ $=15.34]$. The first extinction session was of a priori interest because it contained the first tone presentations following the context switch given Group BT. We therefore performed planned comparisons on data from the first session. These revealed substantially more headjerking in Groups AT and BT than in Group CTRL $[F \mathrm{~s}(1,97)>134.67]$; however, the difference between BT and AT did not approach statistical reliability $[F(1,97)$ $<1]$. The lack of difference was further confirmed by another group $\times$ session ANOVA isolating Groups AT and BT. This analysis revealed a significant session effect $[F(6,84)=63.74]$, indicating extinction, but neither a group effect $[F(1,14)<1]$ nor a group $\times$ session interaction $[F(6,84)<1]$. As in conditioned suppression, switching the context after conditioning had no discernible effect on performance in Group BT.

Headjerking during the pre-CS periods averaged $0.1 \%$, $0.0 \%$, and $0.0 \%$ for Groups AT, BT, and CTRL during this phase. The difference did not approach statistical reliability $[H(2)=2.00]$.

\section{Renewal Testing}

Renewal testing, where Group BT was returned to the conditioning context (Context A) following extinction, is shown at right in Figure 1. The figure suggests that renewed responding did occur, and that it persisted over several sessions. (Data from the fifth test session were lost because of an equipment failure.) A group $\times$ session ANOVA revealed a significant group effect $[F(2,21)=$ $28.70]$, a session effect $[F(4,84)=4.52]$, and a group $\times$ session interaction $[F(8,84)=3.41]$. Planned comparisons on the first session indicated that Group BT responded more than either Group AT or Group CTRL $[F \mathrm{~s}(1,90)>32.09]$. Group AT, which simply received continued extinction trials like those in the previous phase, did not differ from Group CTRL $[F(1,90)=1.62]$. (These comparisons are consistent with similar compari-

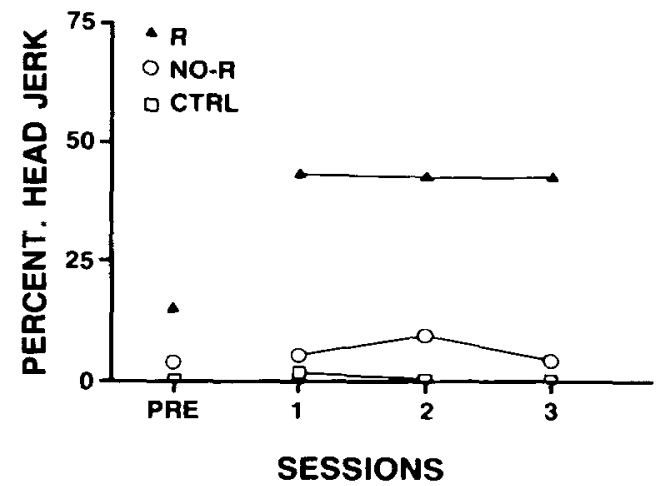

Figure 2. Mean percentage headjerking in response to the tone during reinstatement testing in Experiment 1. The last preceding session in which the tone was nonreinforced in the test context (the extinction context) is shown at left ("pre").

sons that collapsed over sessions.) The results are entirely consistent with prior work in conditioned suppression.

Headjerking during the pre-CS periods averaged $0.1 \%$, $0.0 \%$, and $0.0 \%$ for Groups AT, BT, and CTRL during this phase. These values did not differ $[H(2)=2.00]$. During the first session taken alone, mean headjerking during the pre-CS period was $0.0 \%$ for all groups. Renewed headjerking in Group BT was clearly confined to the CS.

\section{Reinstatement}

Data from the reinstatement test are summarized in Figure 2; they suggest substantial reinstatement of headjerking as a result of exposure to the US in Group R. A group $\times$ session ANOVA on the three test sessions yielded a reliable group effect $[F(2,21)=56.34]$; neither the session effect nor the group $\times$ session interaction was reliable $\left(F_{\mathrm{s}}<1\right)$. Planned comparisons on the first session indicated that Group $\mathbf{R}$ responded significantly more than Group No-R and Group CTRL $[F \mathrm{~s}(1,50)>42.73]$; Groups No-R and CTRL did not differ $[F(1,50)<1]$. The results clearly suggest that US exposures can reinstate extinguished responding in this paradigm, and that they do not increase responding to a control stimulus that has not received excitatory conditioning (Group CTRL).

The groups did not differ in headjerking during the preCS period during reinstatement testing. Groups R, No-R, and CTRL had mean scores of $0.0 \%, 0.3 \%$, and $0.0 \%$ during the test phase, respectively $[H(2)=2.00]$. During the first session, the means were $0.0 \%, 0.8 \%$, and $0.0 \%$ for the same groups $[H(2)=2.00]$. With the procedures used here, headjerking during all phases occurred almost exclusively during the CS.

\section{Discussion}

The results of this experiment are consistent with previous observations in conditioned suppression. First, with the present methods, a change of context immediately following conditioning (Group BT) had no discernible effect on the strength of the conditioned response (cf. Bouton 
\& King, 1983; Bouton \& Swartzentruber, 1986). Second, upon return to the context of conditioning following extinction, Group BT showed a substantial renewal of conditioned responding (cf. Bouton \& Bolles, 1979a; Bouton $\&$ King, 1983). And third, exposure to the US alone following extinction reinstated responding to the CS (e.g., Bouton, 1984; Bouton \& Bolles, 1979b; Bouton \& King, 1983; Rescorla \& Heth, 1975). If there is a difference between the present results and prior conditioned suppression data, it is that renewal and reinstatement appeared to persist over more test trials here. However, we would not propose a general difference between appetitive and defensive conditioning, because of the many parametric differences between the corresponding experiments. For example, the present experiment employed many more conditioning trials (cf. Experiment 2).

The results of the reinstatement "subexperiment" strongly suggest that separate delivery of the US can reinstate extinguished responding in appetitive conditioning. Other details of the experiment also deserve comment. First, the fact that US delivery caused responding to the tone in Group R, but not in Group CTRL (the conditioning control), suggests that reinstatement depends on initial excitatory conditioning to the tone. Second, because US delivery occurred $24 \mathrm{~h}$ prior to the first reinstatement test, headjerking in Group $\mathrm{R}$ was not simply elicited by short-term aftereffects of the US that had become associated with the response during conditioning. And finally, it is worth noting that Group $R$ showed no evidence of headjerking during the pre-CS periods of the test. On the basis of the contextual conditioning account of reinstatement, one might expect some pre-CS responding, because responding to the context is tested alone during the pre-CS periods. Further inspection of the videotapes from reinstatement testing in each of the present experiments also revealed no increase in behaviors directed at the food magazine or in general activity during the preCS periods. The absence of overt evidence of contextual conditioning during these periods is similar to the absence of baseline suppression that is a common finding in conditioned suppression (e.g., Bouton, 1984; Bouton \& Bolles, 1979a; Bouton \& King, 1983, 1986; Rescorla \& Heth, 1975). In either situation, reinstated responding is often confined quite specifically to the CS.

\section{EXPERIMENT 2}

One purpose of the second experiment was to examine renewal and the effect of switching the context after conditioning while controlling for exposure to the two contexts. Context exposure was equated by running the conditioning and extinction phases over two-session "cycles," in which the animal received a session in Context $A$ and a session in Context B. When exposure to A and $B$ is equated in this manner in conditioned suppression, effects like those observed in Experiment 1 still occur (Bouton \& King, 1983, Experiment 2; Bouton \&
Table 1

Design of Experiment 2

\begin{tabular}{lllc}
\hline & & \multicolumn{3}{c}{ Phase } \\
Group & Conditioning & Extinction & Renewal Test \\
\hline AT & AT,+- & AT,-- & AT- \\
BT & AT,+- & BT- - & AT- \\
ATE & AT,+ B- & AT- B- & AT- \\
BTE & AT,+ B- & BT,- A- & AT- \\
\hline
\end{tabular}

Note- $A$ and $B$ refer to contexts; $T=$ tone $C S ;+=$ reinforced trials; $-=$ nonreinforced trials; $-=$ handled in the colony room.

Swartzentruber, 1989, Experiment 2; Lovibond et al., 1984, Experiment 1b).

As illustrated in Table 1 , the design was a $2 \times 2$ factorial which manipulated (a) the context of extinction and (b) whether exposure to the two contexts was equated during conditioning and extinction. Although context exposure has been controlled before (Bouton \& King, 1983; Bouton \& Swartzentruber, 1989; Lovibond et al., 1984), this is the first time it has been manipulated experimentally. When nonreinforced exposures to Context $\mathrm{B}$ are alternated with conditioning sessions in Context A, they might facilitate discrimination of the two contexts, and thereby increase the chances of finding a loss of responding when the tone is switched to Context $\mathrm{B}$ following conditioning (e.g., compare the results of Experiments 1A and $1 B$ in Lovibond et al., 1984). In addition, nonreinforced exposure to Context $A$ alternated with tone extinction sessions in Context B should weaken Context AUS associations that might have been established during conditioning. If the renewal effect depends on the presence of such context-US associations during testing, one would expect exposure to weaken renewal in Group BTE.

As in Experiment 1, all animals entered a reinstatement phase after the conclusion of renewal testing. In the present study, every subject received exposure to the US; half received the US in the context where testing was to occur and half received it in the alternate context. Prior results in conditioned suppression suggest that reinstatement should be stronger when the USs and testing occur in the same context (Bouton, 1984; Bouton \& Bolles, 1979b; Bouton \& King, 1983).

\section{Method}

\section{Subjects and Apparatus}

The subjects were 32 female Wistar rats from the stock that provided those used in Experiment 1. They were approximately 150 days old at the start of the experiment. The housing, maintenance, and apparatus were the same as in Experiment 1.

\section{Procedure}

Context $\mathrm{A}$ again represents the conditioning context, and Context $B$ represents the alternate context. Boxes were completely counterbalanced.

Magazine training and preexposure. On Day 1 , half of the subjects were exposed to the transport box while the other half received approximately $4045-\mathrm{mg}$ Noyes food pellets in the home cage. On Day 2, this treatment was reversed. On Days 3 and 4, magazine training was conducted as in Experiment 1. 
Conditioning. The rats were then randomly assigned to one of two conditions on Day 5. Rats in Condition E, for "exposure" $(n=16)$, received equal exposure to Contexts A and B throughout conditioning and extinction. Rats in the remaining condition, condition "handling"' $(n=16)$, received no exposure to the alternate context during conditioning and extinction, but received a comparable amount of handling in the home colony room. Conditioning took place over six 2-day cycles. Tone-food pairings occurred on Day 1 of each cycle for half the rats in each condition, and on Day 2 for the other half. On these days, every rat received four tones in Context $\mathbf{A}$ that terminated in the onset of the US. Trial timing was identical to that for extinction in Experiment 1. On the alternate days, rats in Condition $\mathrm{E}$ were placed in Context $\mathrm{B}$, where no CSs and USs were delivered; the rats in the handling condition were simply handled twice in the colony room so as to correspond to Condition E's trips in and out of the apparatus.

Extinction. On the day after the conclusion of the last conditioning cycle, the rats in each condition were further divided into two new groups $(n=8)$. The rats in Condition E became Groups ATE and BTE; the rats in the handling condition became Groups AT and BT. A and B designate the context in which each group now received tone extinction. The new groups were counterbalanced on previous session sequence and were matched on performance during conditioning.

As in Experiment 1 (and in our previous conditioned-suppression work), all rats received a 60 -min exposure to the context in which extinction was to be conducted on the 1 st day of the new phase (Day 17). Extinction proper, which began on Day 18, consisted of six 2-day cycles similar to those of conditioning. Now, however, all rats received the tone on Day 1 of each cycle. On Day 2, the rats in Condition $E$ received exposure to the alternate context (Context B for ATE and Context A for Group BTE), whereas Groups $A T$ and $B T$ received equal handling. The extinction sessions contained four nonreinforced tones and were identical to those of Experiment 1 . To summarize, Groups ATE and AT received extinction in the conditioning context, whereas groups BTE and BT received extinction in the alternate context. Exposure to the Contexts A and B was equated in Groups ATE and BTE.

Renewal testing. On the day after the conclusion of the last extinction cycle, 3 days of renewal testing began. All the animals were returned to Context $A$, where they received four daily nonreinforced tests of the CS. The CSs were delivered according to the schedule used during extinction; no USs were ever presented.
Reinstatement. On the next day, all the rats were given eight reinstating USs following the procedure used in Experiment 1. Half the rats in each original group now got USs in the context in which testing was to occur, and half got the USs in the different context. As before, the test context for every animal was the context in which responding had been extinguished during the extinction phase (Context A for members of Groups AT and ATE; B for BT and BTE). There were four final groups. Groups $S$ and SE (from the handling and exposure conditions, respectively) received the reinstating USs in the same context as that of testing. Groups D and DE (from the handling and exposure conditions, respectively) received the USs in the alternate context-that is, one different from that of testing. The two groups from each original exposure condition were matched on extinction performance; as usual, boxes were completely counterbalanced. During each of three daily test sessions that began $24 \mathrm{~h}$ after US presentation, there were four tests of the tone following the usual procedure.

Headjerking was scored throughout the experiment with the methods used in Experiment 1. Interobserver agreement (assessed on the last session of conditioning and on the first and last session of each of the phases that followed) varied little between sessions and averaged $88 \%$. One rat from Group AT had to be eliminated from the experiment due to illness (this rat would also have been assigned to Group D); although this affected the completeness of the counterbalancing, no counterbalanced factor had a systematic effect on the results.

\section{Results}

\section{Conditioning}

Headjerking in response to the tone during each of the six conditioning sessions is shown at left in Figure 3. Conditioning yielded substantial and comparable headjerking in all groups. Some of the data were lost from Session 3 because of an equipment failure, and this session was therefore excluded from statistical analysis (the figure presents the mean of the remaining animals). An exposure $x$ extinction context $\times$ session ANOVA revealed only a significant main effect of session $[F(4,108)=66.69]$. None of the remaining main effects or interactions approached significance (all $F$ s $<1$ ). The groups entered

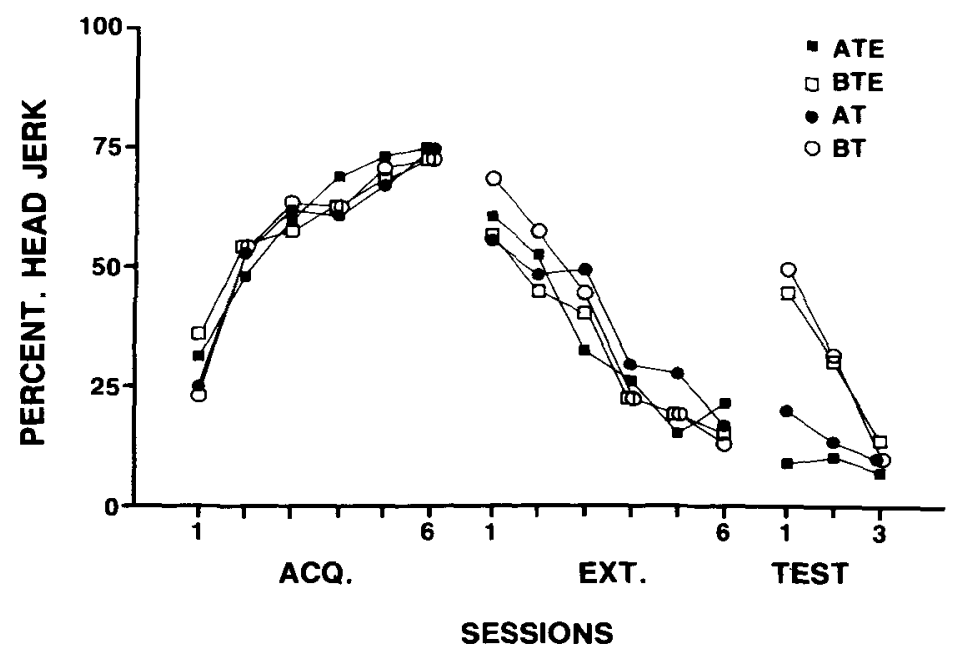

Figure 3. Mean percentage headjerking in response to the tone during the conditioning phase (left), extinction phase (middle), and renewal test phase (right) of Experiment 2. 
the remaining phases with comparable headjerking in response to the CS.

Headjerking during the pre-CS periods of the phase averaged $0.3 \%, 0.3 \%, 0.7 \%$, and $1.0 \%$ for Groups ATE, BTE, AT, and BT, respectively. As in Experiment 1, the response occurred very infrequently outside of the CS, and pre-CS headjerking did not differ significantly among the groups $[H(3)=4.03, p>.10]$.

\section{Extinction}

The results of extinction are presented in the middle portion of Figure 3. Consistent with Experiment 1, there was no evidence that a context switch prior to extinction reduced headjerking in response to the CS. An exposure $x$ context $\times$ session ANOVA revealed only a main effect of session $[F(1,27)=57.00]$; headjerking declined over extinction. No other main effect or interaction, including those associated with the context factor, approached statistical significance (all $F \mathrm{~s}<1$ ). Conditioned responding thus generalized across contexts regardless of the exposure procedure. As usual, headjerking during the pre-CS periods was infrequent (averaging $0.1 \%, 0.0 \%$, $0.0 \%$, and $0.0 \%$ for Groups ATE, BTE, AT, and BT) and did not differ among the groups $[H(3)=2.88$, $p>$.10].

\section{Renewal Testing}

Headjerking in response to the tone during the three sessions of renewal testing is presented at right in Figure 3. Both of the groups that received tone extinction in Context B (Groups BT and BTE) renewed their responding when returned to Context $A$. An exposure $\times$ extinction context $\times$ session ANOVA revealed a reliable main effect of extinction context $[F(1,27)=24.92]$, a session effect $[F(2,54)=25.87]$, and a session $\times$ extinction context interaction $[F(2,54)=12.31]$. No other main effect or interaction approached significance (all $F$ s $<1.10$ ). The renewal effect observed in Experiment 1 was replicated, and the present exposure treatment had no apparent effect on it. No headjerks were scored at any point in any group during the pre-CS periods of this phase.

\section{Reinstatement Testing}

Figure 4 presents headjerking in response to the tone during reinstatement testing. Although all groups appeared to increase their responding from the last preceding tone exposure in the context of testing ("pre"), there was more responding in the groups that received the reinstating USs in the test context (Groups $S$ and SE) than in those that received it in the different context (Groups $D$ and DE). A context $\times$ exposure $\times$ session ANOVA indicated a significant context main effect $[F(1,27)=5.47]$ as well as a session effect $[F(2,54)=12.80]$, and a context $\times$ session interaction $[F(2,54)=3.68]$. No other main effect or interaction was reliable $\left(F_{\mathrm{s}}<1\right)$. Pre-CS headjerking during testing averaged $0.0 \%, 0.0 \%, 0.2 \%$, and $0.0 \%$ for Groups SE, DE, S, and D; the difference did not approach significance $[H(3)=0.37]$. These data replicate

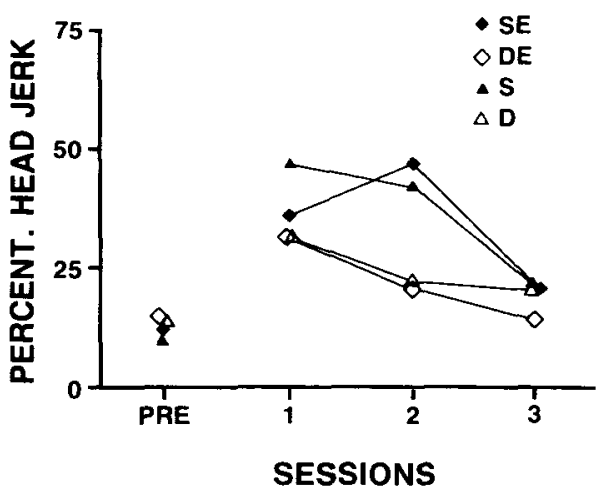

Figure 4. Mean percentage headjerking in response to the tone during reinstatement testing in Experiment 2. The last preceding session in which the tone was nonreinforced in the test context (the extinction context) is shown at left ("pre").

the reinstatement effect obtained in Experiment 1, and they suggest that it depends at least partly on US delivery and testing occurring in the same context.

\section{Discussion}

The results of this experiment extend those of Experiment 1 . As before, the context switch following conditioning had no effect on responding in Group BT, while the return to the conditioning context after extinction renewed extinguished responding to the CS. More important, equating exposure to the two contexts (Groups ATE and BTE) had no appreciable effect on these results. The lack of an effect of exposure on renewal is consistent with the view that renewal does not depend on Context A's direct associations with the US (see also Bouton \& King, 1983; Bouton \& Swartzentruber, 1986); exposure to Context A during extinction should have weakened the A-US association in Group BTE relative to Group BT, but renewal was quite similar in these groups. In addition, Group BTE's nonreinforced exposure to Context B during conditioning should have helped establish a discrimination between $A$ and $B$ prior to extinction; it is interesting that switching the tone to Context B still had no effect on responding to the tone. Neither renewal nor the lack of an effect of the context switch after conditioning was affected by our equation of exposure to the two contexts.

The reinstatement results of this experiment suggest that reinstatement depends at least partly on context; reinstated responding was stronger when US delivery and testing occurred in the same context. This result is consistent with conditioned suppression research (Bouton, 1984; Bouton \& Bolles, 1979b; Bouton \& King, 1983), where the role of contextual conditioning has been directly confirmed by independent evidence of associations to the context (Bouton, 1984; Bouton \& King, 1983). However, unlike prior work on conditioned suppression, presenting the US in the alternate context also appears to have produced an effect; despite apparently complete extinction, Groups D and DE did respond during reinstatement testing. It is important to note, however, that responding in these groups 
might reflect spontaneous recovery resulting from the mere passage of time between extinction and reinstatement testing. This possibility was examined in Experiment 3.

\section{EXPERIMENT 3}

One purpose of the final experiment was to replicate the effect of context in reinstatement while also assessing spontaneous recovery. Another purpose was to examine whether the previous results generalize to a procedure involving massed conditioning and extinction trials. Massed trials were of interest because it would be useful to know whether similar results obtain with procedures that take less laboratory time. And trial massing, like the exposure manipulation examined in Experiment 2, might also influence the strength of context-US associations; for example, massed trials would be expected to produce stronger Context A-US associations during conditioning (cf. Rescorla \& Wagner, 1972). In the present experiment, 40 conditioning trials were administered in a single 2-h session.

\section{Method}

\section{Subjects and Apparatus}

The subjects were 16 male Wistar rats from the same stock as before, approximately 180 days old at the start of the experiment. Housing, maintenance, and apparatus were also the same as in the preceding experiments.

\section{Procedure}

As usual, Context A refers to the conditioning context and Context B to the alternate context; actual boxes were completely counterbalanced.

Magazine training. All rats were initially magazine trained in both contexts. On the 1 st day, the rats were shaped by hand in $30-$ min sessions conducted in each context; the sessions were spaced $1 \mathrm{~h}$ apart. For each session, the magazine was initially baited with two Noyes pellets and an equivalent portion of Wayne Rodent Blox, the maintenance diet. An experimenter watched each rat's behavior via video monitor and occasionally delivered a pellet when the rat was near the magazine. Eight rats were shaped at a time, and an average of eight USs were delivered to each rat in each session. All rats had learned to approach and eat from the magazine at the sound of the feeder click by the end of the second session.

Conditioning. On Day 2, all rats received a 2 -h conditioning session in Context $\mathrm{A}$, in which $\mathbf{4 0}$ tone-food pairings were delivered with a mean ITI of $2.04 \mathrm{~min}$. Each 10-sec tone terminated with the onset of the US.

Extinction. On Day 3, the rats were assigned to two groups in a manner that counterbalanced boxes and the context of initial magazine training; the groups were also matched approximately on performance during conditioning. As usual, Group AT received extinction in Context A, whereas Group BT received it in Context B. Two 1-h extinction sessions were administered on this day. In each session, the rats received 16 tones alone with an average ITI of $3.12 \mathrm{~min}$. The interval between sessions was $4 \mathrm{~h}$, spent in the home cage.

Renewal testing. On Day 4, all the rats were returned to Context A, where they received one 1-h session following the procedure used in extinction. There were 16 tone presentations in each session.
Reinstatement. On the day following renewal testing, the rats were reassigned to three new groups $(n=5)$, with the restriction that no more than 3 rats from an original group or from the same set of physical boxes were assigned to any new group. (One rat was arbitrarily dropped from the experiment to arrange equal $n s$ ). Of the new groups, Group Same and Group Diff each consisted of 3 rats from Group AT and 2 rats from Group BT; Group No-R consisted of 3 rats from BT and 2 from AT.

Each animal's context of extinction was again used as the reinstatement test context. On Day 5, Group Same received eight reinstating USs (following the usual procedure) in the test context, Group Diff received the same treatment in the alternate context, and Group Control received no USs but spent an equal amount of time $(1 \mathrm{~h})$ in the test context. On the following day, all rats received 16 tests of the tone alone (in the test context) following the procedure used in extinction.

The observational procedure was the same as that in the preceding experiments. During conditioning, however, we scored only Trials 1-4, 17-20, and 37-40 (i.e., the first, middle, and last fourtrial blocks). During extinction and renewal testing, we focused on the first and last four-trial blocks of each session (i.e., Trials 1-4 and 13-16); the reinstatement analysis focused on the first eight test trials. Informal observations of the unscored trials suggested that they were consistent with the trials we sampled. On the first and last four trials of conditioning, extinction, and renewal testing, as well as on the first 4 trials of reinstatement testing, a "blind" observer scored 2 rats selected at random from each group ( 1 from each set of boxes). Overall interobserver agreement was $92 \%$.

\section{Results}

\section{Conditioning}

The acquisition of headjerking in response to the tone is shown at left in Figure 5. Not surprisingly, a group $x$ trial-block ANOVA indicated neither a difference between the groups $[F(1,14)<1]$ nor a group $\times$ trial-block interaction $[F(2,28)<1]$, although the trial-block effect was reliable $[F(2,28)=43.34]$. As usual, there was relatively little headjerking during the pre-CS periods, which averaged $1.3 \%$ and $3.3 \%$ for Groups AT and BT over the phase, respectively $[H(1)=1.06]$.

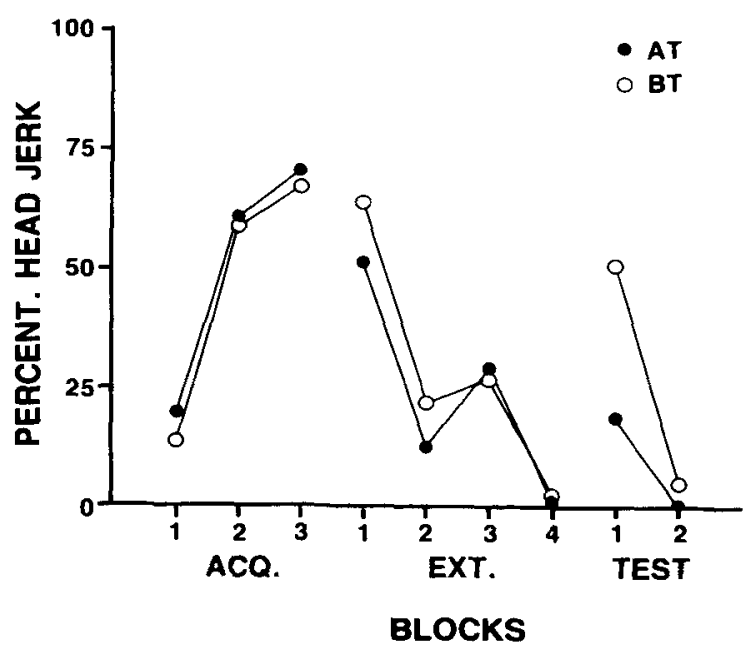

Figure 5. Mean percentage headjerking in response to the tone during the conditioning phase (left), extinction phase (middle), and renewal test phase (right) of Experiment 3. 


\section{Extinction}

The results of extinction, shown in the middle of Figure 5, were highly consistent with the results of the previous experiments. A group $\times$ trial-block ANOVA uncovered neither a difference between the groups $[F(1,14)<1]$, nor a group $\times$ trial-block interaction $[F(3,42)=1.21]$. The trial-block effect was reliable $[F(3,42)=43.44]$. Headjerking during the pre-CS period over the phase averaged $0.8 \%$ and $1.6 \%$ for Groups AT and BT; there was no difference between the groups $[H(1)=0.06]$.

\section{Renewal testing}

The right-hand portion of Figure 5 presents the results of renewal testing. As before, a return to Context $\mathrm{A}$ increased headjerking in Group BT quite substantially. A group $\times$ trial-block ANOVA indicated that the group effect was significant $[F(1,14)=7.69]$, as were the group $\times$ trial-block interaction $[F(1,14)=11.34]$ and the trialblock effect itself $[F(1,14)=56.42]$. Pre-CS headjerking averaged $0.0 \%$ for both groups during the phase.

\section{Reinstatement}

The results of reinstatement testing are summarized in Figure 6. These results were clear, despite the use of small $n \mathrm{~s}(=5)$ in this part of the experiment; original group membership had no effect on the results. A group $\times$ trialblock ANOVA indicated a significant group effect $[F(2,12)=11.37]$. The trial-block effect was also reliable $[F(1,12)=8.87]$, although the group $\times$ trial-block interaction was not $[F(2,12)=1.40]$. Planned comparisons among the groups (collapsing over trial block) revealed a reliable difference between Groups Same and Diff $[F(1,12)=6.01]$, replicating the results of Experiment 2 . However, Groups Same and Diff both exhibited more headjerking than Group No-R, the group that received no USs prior to testing $[F \mathrm{~s}(1,12)=22.73$ and 5.36 , respectively]. Thus, although US presentation in the context of

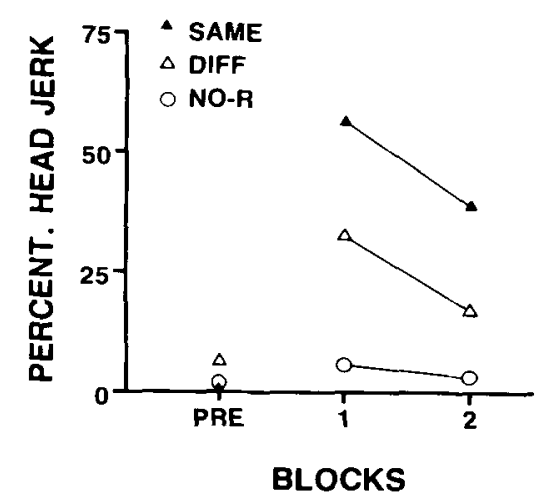

Figure 6. Mean percentage headjerking in response to the tone during reinstatement testing in Experiment 3. The last preceding session in which the tone was nonreinforced in the test context (the extinction context) is shown at left ("pre"). testing resulted in the strongest reinstatement, presentation of the US in a different context did yield some reinstatement. Headjerking during the pre-CS periods of the same trials averaged $0.6 \%, 0.3 \%$, and $0.0 \%$ for Groups Same, Diff, and No-R, respectively, and did not differ reliably $[H(2)=1.09]$.

\section{Discussion}

Despite the use of a more compressed trial schedule (the same number of conditioning trials were delivered here as in Experiment 1, but in one fifth the session time), the results of this experiment were quite consistent with those of Experiments 1 and 2. A context switch following conditioning once again had no significant effect on performance, and a return to Context $A$ following extinction in Context B once again caused a strong renewal of responding in Group BT. Since Context A-US associations should have been especially strong following the present massed-trial conditioning procedure, it is noteworthy that the switch to a neutral context following conditioning still failed to affect performance in Group BT.

The results of the reinstatement test replicate those of Experiment 2 in suggesting that reinstatement is strongest when the US is presented in the test context. However, they further suggest that some reinstatement may occur even when the US is delivered in a different context; during reinstatement testing, Group Diff responded significantly more than a control that received no exposure to the US (Group No-R). In contrast, we have not observed any effect of US delivery in a different context in conditioned suppression studies of reinstatement (Bouton, 1984; Bouton \& Bolles, 1979b; Bouton \& King, 1983, 1986). This possible difference between the two conditioning preparations would stand as an exception to a strong set of parallels that the present experiments have otherwise begun to establish.

\section{GENERAL DISCUSSION}

The present experiments extend the generality of several phenomena from conditioned suppression to appetitive conditioning. With the appetitive procedures used here, (1) a context switch immediately after conditioning failed to affect performance to the CS; (2) a return of the CS to the context of conditioning following extinction in another context consistently renewed extinguished responding; and (3) presentation of the US alone following extinction reinstated extinguished responding, especially when it was presented in the context where the CS was subsequently tested (Experiments 2 and 3 ). Each of these results was obtained over a range of procedures. Experiments 1 and 2 included widely spaced conditioning and extinction trials; Experiment 3 involved relatively massed conditioning and extinction trials; and in Experiment 2 , the procedure intermixed and equated exposure to Contexts A and B throughout conditioning and extinc- 
tion. All together, the data suggest some generality for the present results as well as commonality between appetitive and aversive conditioning.

The similarity between the results of the two conditioning preparations encourages the view that similar mechanisms may operate within them. In principle, renewal can be explained by assuming that Context $A$ becomes excitatory during the conditioning phase and/or that Context $B$ becomes inhibitory during extinction; either type of context-US association would determine performance by summating with associative strength of the CS. However, previous research has produced little evidence to support this hypothesis. In conditioned suppression, demonstrable context-US associations are not necessary for a context to affect CS performance (Bouton \& King, 1983; Bouton \& Swartzentruber, 1986); conversely, strong and demonstrable associations to the context are often not sufficient to affect CS performance either (Bouton, 1984; Bouton \& King, 1986). Although Lovibond et al. (1984, Experiments $1 \mathrm{C}$ and 2) presented data that they claimed supported an associative interpretation of renewal, their results, like the renewal effect itself, are consistent with either an associative interpretation or the view that contexts set the occasion for the CS-US association (see discussion in Bouton \& Swartzentruber, 1986, p. 349). There is currently little direct evidence to support the inference that renewal is produced by excitatory context-US associations to Context A or inhibitory context-US associations to Context B.

Instead, as suggested above, contexts may affect CS performance by retrieving or setting the occasion for whole CS-US associations (Bouton \& Bolles, 1985; Bouton \& Swartzentruber, 1986; cf. Holland, 1985; Rescorla, 1985). According to this view, renewal may be controlled by Context A's signaling or retrieving the CS-US relation and/or Context B's signaling CS-no US. In reinstatement, where performance does appear to be affected by direct context-US associations (e.g., Bouton, 1984; Bouton \& King, 1983, 1986), context excitation may itself retrieve the original CS-US association instead of simply summating with excitation to the CS (Bouton \& King, 1986; see also Kaplan \& Hearst, 1985). The present results are compatible with such mechanisms. For example, the view that the renewal effect is not a product of CS-context summation is consistent with the results of Experiment 2, which suggest that renewal was not affected by extinction exposure to Context $A$ that should have reduced its associative strength during testing. Occasion setters are often not affected by extinction (Rescorla, 1985). In addition, if one assumes that Context A does acquire some excitatory strength during conditioning (see especially Experiment 3), the summation view might not anticipate our consistent failure to observe an effect of switching the CS to a different, associatively neutral context after conditioning. As in conditioned suppression, however, such observations do not imply that context-US associations cannot affect responding under some circumstances. The present data suggesting a role of context in reinstatement are consistent with the view that con- textual excitation created by US exposure can enhance responding to a $C S$ that has been extinguished. ${ }^{I}$

The finding that some reinstatement did occur when USs were delivered in a different context (Experiment 3; see also Experiment 2) contrasts with prior conditionedsuppression data. There are several possible reasons for the difference in results. First, there may have been some generalization between the present contexts, which were (1) provided by different boxes than those used in the conditioned suppression experiments and (2) both initially associated with the US during magazine training, unlike the contexts used in aversive conditioning. However, differences in generalization seem unlikely, given the otherwise consistent results; it is not clear why renewal should be so robust if the animals generalized substantially between the present contexts. Second, unlike the present food US, the footshock used in conditioned suppression is a highly novel event that is delivered through a physical feature of the context (the grid floor). This might encourage the rat to encode footshock as a feature of a specific context during reinstatement. On the other hand, context-specific encoding may not be consistent with the fact that fear of a CS based on footshock generalizes relatively well across contexts. Finally, even though the present apparatus was cleaned after every session, it is probably impossible to eliminate lingering food odors. Thus, the odor of food may have persisted beyond the session in which it was delivered, until reinstatement testing on the next day. A food odor common to the USdelivery and test contexts could have helped restore responding in Group Diff (recall that the contexts were counterbalanced). Note that the odor's recent association with food delivery would be necessary for it to reinstate responding; rats not given reinstating USs did not respond the next day, despite being tested in the presence of the same odor (Experiments 1 and 3). In effect, a food odor would provide another contextual cue mediating reinstatement because of its recent association with the US.

Prior research on the contextual control of appetitive conditioning has often assumed competition between the context and CS in generating performance (e.g., Gibbon \& Balsam, 1981; Jenkins, Barnes, \& Barrera, 1981). Like similar observations in aversive conditioning, the present observation of reinstatement, for example, may suggest that contextual conditioning may also facilitate, rather than compete with, responding to the CS. The present results are consistent with a larger set of data from aversive conditioning in suggesting that the context is especially likely to facilitate CS responding after an extinction treatment, which may generate ambiguity in the meaning of the CS.

\section{REFERENCES}

Balsam, P. D., SChwartz, A. L. (1981). Rapid contextual conditioning in autoshaping. Journal of Experimental Psychology: Animal Behavior Processes, 7, 382-393.

Bouton, M. E. (1984). Differential control by context in the inflation and reinstatement paradigms. Joumal of Experimental Psychology: Animal Behavior Processes, 10, 56-74.

Bouton, M. E. (1988). Context and ambiguity in the extinction of emo- 
tional learning: Implications for exposure therapy. Behaviour Research \& Therapy, 26, 137-149.

Bouton, M. E., \& Bolles, R. C. (1979a). Contextual control of the extinction of conditioned fear. Learning \& Motivation, 10, 445-466.

Bouton, M. E., \& Bolles, R. C. (1979b). Role of conditioned contextual stimuli in reinstatement of extinguished fear. Journal of Experimental Psychology: Animal Behavior Processes, 5, 368-378.

Bouton, M. E., \& Bolles, R. C. (1985). Contexts, event-memories, and extinction. In P. D. Balsam \& A. Tomie (Eds.), Context and learning (pp. 133-166). Hillsdale, NJ: Erlbaum.

Bouton, M. E., \& King, D. A. (1983). Contextual control of the extinction of conditioned fear: Tests for the associative value of the context. Joumal of Experimental Psychology: Animal Behavior Processes, 9, 248-265.

Bouton, M. E., \& King, D. A. (1986). Effect of context on performance to conditioned stimuli with mixed histories of reinforcement and nonreinforcement. Journal of Experimental Psychology: Animal Behavior Processes, 12, 4-15.

Bouton, M. E., \& Swartzentruber, D. (1986). Analysis of the associative and occasion-setting properties of contexts participating in a Pavlovian discrimination. Journal of Experimental Psychology: Animal Behavior Processes, 12, 333-350.

Bouton, M. E., \& SWartzentruber, D. (1989). Slow reacquisition following extinction: Context, encoding, and retrieval mechanisms. Joumal of Experimental Psychology: Animal Behavior Processes, 15, 43-53.

Channell, S., \& Hall, G. (1983). Contextual effects in latent inhibition with an appetitive conditioning procedure. Animal Learning \& Behavior, 11, 67-74.

Gibbon, J., \& Balsam, P. (1981). Spreading association in time. In C. M. Locurto, H. S. Terrace, \& J. Gibbon (Eds.), Autoshaping and conditioning theory (pp. 219-253). New York: Academic Press.

GrAU, J. W., \& RESCORLA, R. A. (1984). Role of context in autoshaping. Journal of Experimental Psychology: Animal Behavior Processes, 10, 324-332.

Hanford, P. V., Mulvaney, D. E., \& Kelfer, D. A. (1980). The effect of novel environments on CS extinction in a conditioned suppression paradigm. Bulletin of the Psychonomic Society, 16, 341-344.

Holland, P. C. (1977). Conditioned stimulus as a determinant of the form of the Pavlovian conditioned response. Journal of Experimental Psychology: Animal Behavior Processes, 3, 77-104.

Holland, P. C. (1979). The effects of qualitative and quantitative variation in the US on individual components of Pavlovian appetitive conditioned behavior in rats. Animal Learning \& Behavior, 7, 424-432.

Holland, P. C. (1985). The nature of conditioned inhibition in serial and simultaneous feature negative discriminations. In R. R. Miller \& N. E. Spear (Eds.), Information processing in animals: Conditioned inhibition (pp. 267-297). Hillsdale, NJ: Erlbaum.

Howel., D. C. (1982). Statistical methods for psychology. Boston: Duxbury Press.

Jenkins, H. M., Barnes, R. A., \& Barrera, F. J. (1981). Why autoshaping depends on trial spacing. In C. M. Locurto, H. S. Terrace, \& J. Gibbon (Eds.), Autoshaping and conditioning theory. New York: Academic Press.

Kaplan, P. S., \& Hearst, E. (1985). Contextual control and excitatory versus inhibitory learning: Studies of extinction, reinstatement, and interference. In P. D. Balsam \& A. Tomie (Eds.), Context and learning (pp. 195-224). Hillsdale, NJ: Erlbaum.

Lovibond, P. F., Preston, G. C., \& Mackintosh, N. J. (1984). Context specificity of conditioning, extinction, and latent inhibition. Journal of Experimental Psychology: Animal Behavior Processes, 10, 360-375.

Rescorla, R. A. (1985). Conditioned inhibition and facilitation. In R. R. Miller \& N. E. Spear (Eds.), Information processing in animals: Conditioned inhibition (pp. 299-326). Hillsdale, NJ: Erlbaum.

Rescorla, R. A., Heth, C. D. (1975). Reinstatement of fear to an extinguished conditioned stimulus. Journal of Experimental Psychology: Animal Behavior Processes, 1, 88-96.

Rescorla, R. A., \& Wagner, A. R. (1972). A theory of Pavlovian conditioning: Variations in the effectiveness of reinforcement and nonreinforcement. In A. H. Black \& W. F. Prokasy (Eds.), Classical conditioning II: Current research and theory (pp. 64-99). New York: Appleton-Century-Crofts.

TомIE, A. (1976). Interference with autoshaping by prior context conditioning. Journal of Experimental Psychology: Animal Behavior Processes, 2, 323-334.

\section{NOTE}

1. Note that the presence of contextual excitation was inferred rather than directly demonstrated, because we saw no evidence of it in performance during the pre-CS periods of reinstatement testing. It is often difficult to detect contextual excitation in pre-CS behavior, however. In conditioned suppression, contextual excitation in reinstatement has been confirmed with context preference tests conducted at the outset of the test sessions (Bouton, 1984; Bouton \& King, 1983, 1986). Such tests appear to be more sensitive than more direct measures of performance immediately prior to the CS.

(Manuscript received May 2, 1988; revision accepted for publication September 29, 1988.) 